\title{
An investigation of methylation pattern changes in the IKZF1 promoter in patients with childhood B-cell acute lymphoblastic leukemia
}

\author{
Mina Rahmani ${ }^{1}$, Masoumeh Fardi ${ }^{1,2}$, Majid Farshdousti Hagh ${ }^{3}$, Abbas Ali Hosseinpour Feizi ${ }^{4}$, \\ Mehdi Talebi ${ }^{5}$, Saeed Solali ${ }^{6,7}$ \\ ${ }^{1}$ Immunology Research Center, ${ }^{2}$ Student Research Committee, ${ }^{3}$ Drug Applied Research Center, ${ }^{4}$ Hematology and Oncology Research \\ Center, ${ }^{5}$ Immunology Department, ${ }^{6}$ Molecular Medicine Research Center, ${ }^{7}$ Department of Immunology, Division of Hematology and \\ Transfusion Medicine, Faculty of Medicine, Tabriz University of Medical Sciences, Tabriz, Iran
}

p-ISSN 2287-979X / e-ISSN 2288-0011

https://doi.org/10.5045/br.2019.54.2.144

Blood Res 2019;54:144-148.

Received on December 21, 2018

Revised on March 19, 2019

Accepted on April 4, 2019

\section{Background}

Ikaros family zinc finger 1 (IKZF1)is a transcription factor with an important role in controlling hematopoietic proliferation and function, particularly lymphoid cell differentiation. It was previously shown that various mechanisms and expression patterns of Ikaros are linked to a variety of cancers. We hypothesized that aberrant methylation (hypomethylation) of the IKZF1 promoter region might be one of the causes of B-cell acute lymphoblastic leukemia (B-ALL). In B-ALL patients, an increased expression of this gene is a potential cause of B-cell differentiation arrest and proliferation induction. Therefore, as more than $90 \%$ of patients with ALL are $<15$ years old, we investigated the methylation pattern of the IKZF1 promoter in childhood B-ALL.

\section{Methods}

Twenty-five newly diagnosed B-ALL cases were included (all younger than $15 \mathrm{yr}$ ). In addition, we selected 25 healthy age- and sex-matched children as the control group. We collected the blood samples in EDTA-containing tubes and isolated lymphocytes from whole blood using Ficoll 1.077 Lymphosep. Next, we extracted genomic DNA with the phenol/chloroform method. Two microgram of DNA per sample was treated with sodium bisulfite using the EpiTect Bisulfite Kit, followed by an assessment of DNA methylation by polymerase chain reaction (PCR) analysis of the bisulfite-modified genomic DNA.

\section{Results}

Our data highlighted a hypomethylated status of the IKZF1 promoter in the ALL cases (96\% of the cases were unmethylated). In contrast, the control group samples were partially methylated (68\%).

\section{Conclusion}

This study demonstrated a hypomethylated pattern of the IKZF1 promoter region in childhood B-ALL, which might underlie the aberrant Ikaros expression patterns that were previously linked to this malignancy.

Key Words Leukemia, Ikaros, IKZF1, DNA methylation, Hematological malignancy

\section{INTRODUCTION}

Acute leukemia is the most common pediatric malignancy and accounts for approximately $29 \%$ of all childhood malignancies. Continuing along this line, acute lymphoblastic leukemia (ALL) accounts for approximately $76 \%$ of acute leukemia in children, while the subtype called B-cell ALL (B-ALL) constitutes more than $80 \%$ of ALL cases in children [1]. B-ALL is a malignant clonal tumor originating from a hematopoietic stem cell with chromosomal aberrations. It is associated with massive proliferation and accumulation of lymphoblasts that exhibit maturation arrest at the early stages of differentiating into lymphocytes. Furthermore, the 
disease has been associated with a wide range of molecular and clinical changes that might aggravate disease progression [2]. These changes include epigenetic alterations such as DNA methylation, which play a key role in regulating gene expression. This DNA methylation mechanism is characterized by the addition of a methyl group to the 5' position of the cytosine ring in cytosine-phosphate diester-guanine $(\mathrm{CpG})$ islands $[3,4]$.

Ikaros is a transcription factor of the zinc finger family encoded by the Ikaros family zinc finger 1 (IKZF1) gene and it is crucial in regulating lymphoid development and immunoglobulin rearrangement. Although Ikaros plays an important role in the normal development of the immune system, excessive has been linked to various cancers, such as bladder, breast, blood, glial, head and neck cancers. This highlights the multi-dimensional character of Ikaros [5] and its role in the initiation and progression of several cancer types [6]. In addition, aberrant expression of Ikaros affects the prognosis of several unique cancer types [5]. Ikaros is primarily involved in ALL biology by attenuating the normal repression of target genes and inhibiting the regulation of genes expression involved in lymphoid differentiation. However, the intricacies of the molecular mechanisms underlying the functionality of Ikaros remain unclear [7].

Although a large number of molecular and genetic studies have explored the pathogenesis and biology of lymphoid malignancies, a detailed analysis of DNA methylation patterns might help explain the mechanisms underlying such diseases [8]. As stated above, Ikaros has a significant role in the incidence of cancers, especially B-ALL. Altered expression levels of IKZF1 and aberrant DNA methylation patterns have been associated with different types of leukemia [9]. Notably, lymphoid B-cell differentiation is disrupted in B-ALL, and IKZF1 is a major regulator of B-cell differentiation. Thus, we hypothesized that aberrant methylation (hypomethylation) of the IKZF1 promoter region results in excessive IKZF1 expression. Overexpression of IKZF1 is a potential cause of $\mathrm{B}$-cell differentiation arrest and proliferation induction in B-ALL patients. Therefore, to further elucidate the role of IKZF1 in B-ALL, we investigated the methylation status of $\mathrm{CpG}$ islands in the promoter region in children who were recently diagnosed with B-ALL.

\section{MATERIALS AND METHODS}

\section{Patient information}

We included 25 cases with recently diagnosed ALL and an age of 15 years or younger. Ninety percent of the patients were in the 1-9 years group, whereas only $10 \%$ were part of the other two age groups. A similar age distribution was observed for the 25 age- and gender-matched controls. According to the complete blood count (CBC) and clinical history, all of these control children were healthy. Diagnosis of all patients, who were in a pre-therapy state, was suggested and confirmed according to clinical, biochemical, morphological and flow cytometry findings made by the same medi- cal doctor. We collected $2 \mathrm{~mL}$ of peripheral blood per subject in ethylenediaminetetraacetic acid (EDTA)-containing tubes. Patient blood samples were collected in the Pediatric Hospital of Tabriz, while blood samples of the control group were collected in the Dey Clinical Laboratory up to one year after having obtained informed consent from the parents. The study was supervised by the Medical Ethics Committee of Tabriz University of Medical Sciences, Iran, under the code 5/4/6065 in 2015.

\section{Lymphocyte separation and DNA extraction}

We isolated lymphocytes from whole blood with Ficoll 1.077 Lymphosep (Biowest, Nuaillé, France), followed by phosphate-buffered saline (PBS) multiple washes. Next, genomic DNA was extracted using the phenol/chloroform method [10]. In turn, extracted DNA was dissolved in 20 $\mu \mathrm{L}$ of RNase- and DNase-free water and frozen $\left(-80^{\circ} \mathrm{C}\right)$ until required. We measured DNA concentrations using a NanoDrop 2000c spectrophotometer (Thermo Fisher Scientific, Lenexa, KS, USA).

\section{Sodium bisulfite treatment}

Of each sample, we treated $2 \mu \mathrm{g}$ of DNA with sodium bisulfite from the EpiTect Bisulfite Kit (QIAGEN, Hilden, Germany), according to the manufacturer's instructions. After this procedure, the processed DNA samples were dissolved in $20 \mu \mathrm{L}$ of elution buffer and stored at $-70^{\circ} \mathrm{C}$. A whole-genome amplified DNA sample, where all CpG sites were unmethylated, and a DNA sample treated with SssI methyltransferase (New England BioLabs, Beverly, MA, USA) (i.e. all CpG sites were methylated) were used as negative and positive controls, respectively [11].

\section{DNA methylation analysis}

We assessed DNA methylation by polymerase chain reaction (PCR) analysis of bisulfite-modified genomic DNA. $150 \mu \mathrm{g}$ treated DNA per sample was utilized for methylation-specific PCR (MS-PCR) and the total PCR reaction volume was $25 \mu \mathrm{L}$. We designed the forward and reverse primers for methylated and unmethylated statuses with MethPrimer (The Li Lab, China) (Table 1).

\section{Statistical analysis}

We used a chi-square test to compare the abundances of the three gene methylation statuses (methylated, unmethylated and partial methylation) using SPSS statistical software version 16.0 (SPSS Inc., Chicago, IL, USA). Chi-square and $t$-tests were used to compare between age and gender. Statistical significance was set at $P<0.05$.

\section{RESULTS}

The patient and control group age medians were 6.5 and 4.5 years, respectively $(P=0.077)$. In addition, the two groups were appropriately matched in terms of sex and there was no significant difference between the two groups $(P=0.08)$. 
Table 1. List of primer pairs used for MS-PCR.

\begin{tabular}{llcc}
\hline \multicolumn{1}{c}{ Primer } & \multicolumn{1}{c}{ Sequence $\left(5 \rightarrow 3^{\prime}\right)$} & Annealing temperature $\left({ }^{\circ} \mathrm{C}\right)$ & Product size (length in bps) \\
\hline IKZF1- methylated forward primer & TTTGTATCGGAGTAGCGATTCG & 59.5 & 200 \\
IKZF1- methylated reverse primer & CTACCGCCCAAAACGACG & 59.5 & 200 \\
IKZF1- unmethylated forward primer & GTATTGGAGTAGTGATTTGGGAG & 57.4 & 199 \\
IKZF1- unmethylated reverse primer & CAACTACCACCCAAACAACA & 57.4 & 199 \\
\hline
\end{tabular}

Table 2. Clinical and laboratory data of control and patient groups.

\begin{tabular}{lcc}
\multicolumn{1}{c}{ Clinical data (units) } & Control group & Patient group \\
\hline $\mathrm{N}$ of samples & 25 & 25 \\
$\mathrm{M} / \mathrm{F}$ ratio & $16 / 9$ & $15 / 10$ \\
Mean age and range $(\mathrm{yr})$ & $4.5(3-12)$ & $6.5(1-14)$ \\
Blood transfusion & - & 2 \\
Cytogenetic & - & 1 case: Down syndrome (trisomy 21) \\
Laboratory data (units) & $12.5(10.214 .4)$ & $7.7(2.9-12.4)$ \\
Mean Hgb and range $(\mathrm{g} / \mathrm{L})$ & $8,405(6,752-10,058)$ & $71,576(38,250-104,902)$ \\
Mean WBC and range $\left(\times 10^{9} / \mathrm{L}\right)$ & $298.640(130,000-622,000)$ & $59.960(3,000-165,000)$ \\
Mean PLT and range $\left(\times 10^{9} / \mathrm{L}\right)$ & - & $75 \%$ of patients \\
LDH more than $800 \mathrm{U} / \mathrm{L}$ & &
\end{tabular}

Clinical characteristics are shown in Table 2.

The principle of the MS-PCR assay is based on the conversion of cytosine $(\mathrm{C})$ to uracil $(\mathrm{U})$ via single base substitution (SBS), when the $\mathrm{C}$ in $\mathrm{CpG}$ is unmethylated. Contrastingly, when the $\mathrm{C}$ in $\mathrm{CpG}$ is methylated, then SBS has no effect on the methylated cytosine. Therefore, following bisulfite modification, two specific primers (targeting unmethylated and methylated states) were used in MS-PCR assay. To assess promoter methylation status via PCR, we designed our primers to specifically amplify methylated or unmethylated sequences in the CpG islands of the IKZF1 promoter. Amplification with the methylated or unmethylated primers resulted in the presence or absence of PCR product, depending on the methylation status of the CpG dinucleotides assayed by primers. We did not detect IKZF1 DNA methylation in the ALL cases (24 patients out of 25 ALL cases or $96 \%$ of the cases were unmethylated), while in the control group two subjects out of 25 (8\%) were methylated, six out of 25 controls (24\%) were unmethylated and 17 out of 25 controls (68\%) were partially methylated. DNA methylation patterns of IKZF1 are presented in Fig. 1.

As shown in Figs. 1, 2, we observed hypomethylation of the IKZF1 gene promoter in patients. Furthermore, in the control group, partial methylation was observed in most cases, while we observed complete methylation in only a few cases.

\section{DISCUSSION}

IKZF1, located at 7p12, is essential for B-cell lineage development. IKZF1 biology is complex. In 1992, Ikaros

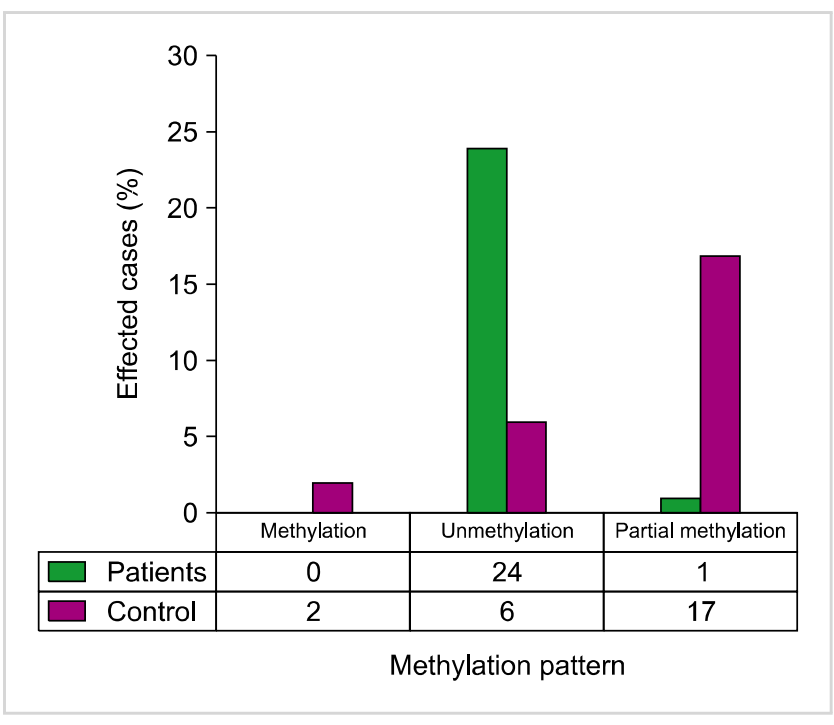

Fig. 1. Methylation patterns differed significantly between the patient and control groups. As shown in this figure, 24 out of 25 patients with B-ALL had an unmethylated pattern in their IKZF1 promoter genomic region, while this was 6 out of 25 normal cases. It shows a significant $(P<0.05)$ unmethylation pattern in our patients with childhood B-ALL. On the other hand, it shows that most normal cases have methylated or partially methylated statues in their IKZF1 gene promoter site.

was identified as a zinc finger protein with an important role in the development and maturation of T-lymphocytes. Ikaros can activate or inhibit target genes' expression by binding to their regulatory regions and subsequently recruiting chromatin modifier complexes, thus initiating differentiation and inhibiting proliferation [12, 13]. Furthermore, Ikaros has been extensively implicated in regulating the de- 


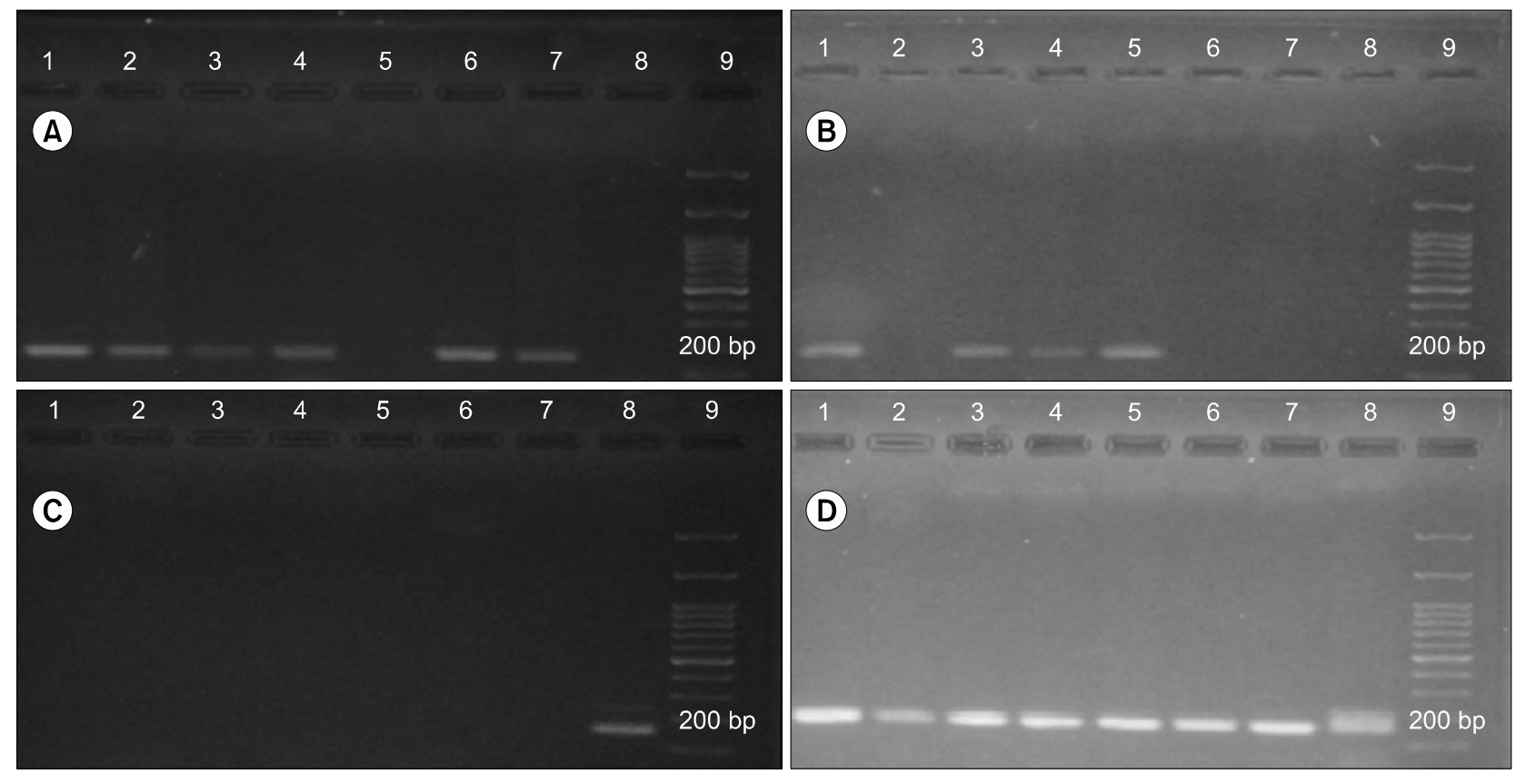

Fig. 2. PCR products were subjected to electrophoresis. IKZF1-associated bands are visible at the 200 bp marker. (A) MSP results with IKZF1 unmethylated primers for the control group. As can be seen, in most of the control samples, the unmethylated pattern was observed. (B) MSP results with the methylated primers of the IKZF1 gene for the control group. As shown in this figure, the majority of these samples exhibited a relative methylation state. (C) MSP results with IKZF1-specific methylated primer for the patient group. As can be seen, samples from patients didn't have any band with methylated primer. (D) MSP results with IKZF1 unmethylated primers for the patient group. All samples of the patients group had recognizable bands with the unmethylated primers of IKZF1 promoter.

velopment of the immune system [14-16]. In addition, some studies have attributed the role of tumor suppression to this transcription factor [5]. Therefore, the deletion or aberrant methylation of the IKZF1 promoter, which results in gene silencing, has been associated with a wide variety of malignancies. Conversely, other studies have shown that an increased expression of the gene is also related to cancers such as leukemia, bladder, lung, and glioma $[5,7]$. In general, it appears that the optimal expression levels of Ikaros vary per tissue and disease condition. Moreover, its deletion or overexpression may contribute to the development of various cancer types. We hypothesized that aberrant methylation (hypomethylation) of the IKZF1 promoter increases IKZF1 expression, and that excessive expression of IKZF1 is a potential cause of B-cell differentiation arrest and proliferation induction in B-ALL patients. Considering the above, we investigated the methylation pattern of the IKZF1 promoter in childhood B-ALL.

Durchdewald et al. [6] reported that FOS family members, including c-Fos, GATA-1, Elk-1, and NKX6-B, have a binding site upstream of the IKZF1 transcription site. These family members dimerize with c-Jun and form AP-1; an important transcription factor in the initiation and progression of cancer.

Although aberrant DNA methylation is a common phenomenon in child and adult ALL patients, it appears that Ikaros gene silencing through this mechanism remains a rare phenomenon in ALL [17]. Our study supports these findings, as we observed a hypomethylation pattern of the
IKZF1 gene promoter in $96 \%$ of our B-ALL samples. It is possible that this hypomethylation might increase the expression of Ikaros in B-ALL patients. This hypothesis is supported by a publication of Zhang et al. [7], which demonstrated an increased expression of Ikaros gene, due to increased gene demethylation, in lung cancer patients.

Ikaros has 8 different exons that encode 8 different isoforms [18]. Long isoforms of this protein (IK1 to 3), with over 3 zinc fingers at their $\mathrm{N}$ terminal, are functional and have a high DNA-binding affinity. However, the short isoforms (IK4 to 8), which possess fewer zinc fingers, have a lower binding affinity and are less competent at transcribing the target genes because they dimerize with the longer isoforms [19-22]. A previous study demonstrated that IK6 expression decreases apoptosis and enhances cell survival, while being associated with B-cell inhibition, differentiation, and maturation in leukemogenesis. Consequently, it was shown that IK6 is the most commonly increased isoform in B-ALL, T-cell ALL (T-ALL) and chronic myeloid leukemia (CML) [23]. Building on this, Han et al. [24] demonstrated that Dominant-negative (DN) isoforms, particularly IK6, are associated with poor prognoses in ALL patients. Furthermore, overexpression of this isoform is known to result in overproliferation and chemotherapy resistance in ALL cell lines. Other studies have shown an increased IK6 cell survival following acetylation of the $\mathrm{Bcl}-\mathrm{xL}$ promoter, a mechanism that was also previously linked to the incidence of T-ALL [20] and pituitary tumors [25]. Therefore, an alternative hy- 
pothesis to the hypomethylation of the IKZF1 promoter and the subsequent increase in Ikaros expression in B-ALL patients is possible. More specifically, hypomethylation in the promoters of the short and $\mathrm{DN}$ protein isoforms is also a plausible mechanism. Thus, further research at the mRNA and protein levels is required.

The results of this study, in conjunction with additional advanced molecular research, might reveal the molecular mechanisms that underlie Ikaros gene in the development of cancers, paticularly B-ALL. Such explorations could pioneer the development of novel therapeutic strategies to treat cancer.

In this study we report a marked hypomethylation of the IKZF1 promoter region in B-ALL pediatric patients. It is plausible that the unmethylated status of the IKZF1 promoter might increase the expression of Ikaros, which has been linked to B-cells differentiation arrest and proliferation induction in B-ALL patients. Thus, targeting and suppressing the excessive expression of IKZF1 is a promising therapy in B-ALL patients.

\section{Authors' Disclosures of Potential Conflicts of Interest}

No potential conflicts of interest relevant to this article were reported.

\section{REFERENCES}

1. Siegel RL, Miller KD, Jemal A. Cancer statistics, 2017. CA Cancer J Clin 2017;67:7-30.

2. Cobaleda C, Sánchez-García I. B-cell acute lymphoblastic leukaemia: towards understanding its cellular origin. Bioessays 2009;31:600-9.

3. Morales-Nebreda L, McLafferty FS, Singer BD. DNA methylation as a transcriptional regulator of the immune system. Transl Res 2018;204:1-18.

4. Fardi M, Solali S, Farshdousti Hagh M. Epigenetic mechanisms as a new approach in cancer treatment: An updated review. Genes Dis 2018;5:304-11.

5. Yang L, Luo Y, Wei J. Integrative genomic analyses on Ikaros and its expression related to solid cancer prognosis. Oncol Rep 2010;24:571-7.

6. Durchdewald M, Angel P, Hess J. The transcription factor Fos: a Janus-type regulator in health and disease. Histol Histopathol 2009;24:1451-61.

7. Zhang Z, Xu Z, Wang X, et al. Ectopic Ikaros expression positively correlates with lung cancer progression. Anat Rec (Hoboken) 2013;296:907-13.

8. Jiang Y, Hatzi K, Shaknovich R. Mechanisms of epigenetic deregulation in lymphoid neoplasms. Blood 2013;121:4271-9.

9. Zhao X, Zhang W, Wang L, Zhao WL. Genetic methylation and lymphoid malignancies: biomarkers of tumor progression and targeted therapy. Biomark Res 2013;1:24.
10. Ozmen F, Ozmen MM, Ozalp N, Akar N. The prevalence of factor V (G1691A), MTHFR (C677T) and PT (G20210A) gene mutations in arterial thrombosis. Ulus Travma Acil Cerrahi Derg 2009;15: 113-9.

11. Milani L, Lundmark A, Kiialainen A, et al. DNA methylation for subtype classification and prediction of treatment outcome in patients with childhood acute lymphoblastic leukemia. Blood 2010;115:1214-25.

12. Lopes BA, Meyer C, Barbosa TC, et al. COBL is a novel hotspot for IKZF1 deletions in childhood acute lymphoblastic leukemia. Oncotarget 2016;7:53064-73.

13. Olsson L, Albitar F, Castor A, et al. Cooperative genetic changes in pediatric B-cell precursor acute lymphoblastic leukemia with deletions or mutations of IKZF1. Genes Chromosomes Cancer 2015;54:315-25.

14. Perotti EA, Georgopoulos K, Yoshida T. An Ikaros promoter element with dual epigenetic and transcriptional activities. PLoS One 2015;10:e0131568.

15. Merkenschlager M. Ikaros in immune receptor signaling, lymphocyte differentiation, and function. FEBS Lett 2010;584: 4910-4.

16. John LB, Ward AC. The Ikaros gene family: transcriptional regulators of hematopoiesis and immunity. Mol Immunol 2011; 48:1272-8.

17. Fang Z, Kuang S, Yang H, Garcia-Manero G. Lack of IKZF1 aberrant DNA methylation in acute lymphocytic leukemia. Blood 2009;114:982.

18. Sun L, Heerema N, Crotty L, et al. Expression of dominantnegative and mutant isoforms of the antileukemic transcription factor Ikaros in infant acute lymphoblastic leukemia. Proc Natl Acad Sci U S A 1999;96:680-5.

19. Nishii K, Katayama N, Miwa H, et al. Non-DNA-binding Ikaros isoform gene expressed in adult B-precursor acute lymphoblastic leukemia. Leukemia 2002;16:1285-92.

20. Meleshko AN, Movchan LV, Belevtsev MV, Savitskaja TV. Relative expression of different Ikaros isoforms in childhood acute leukemia. Blood Cells Mol Dis 2008;41:278-83.

21. Georgopoulos K, Moore DD, Derfler B. Ikaros, an early lymphoid-specific transcription factor and a putative mediator for T cell commitment. Science 1992;258:808-12.

22. Sun L, Liu A, Georgopoulos K. Zinc finger-mediated protein interactions modulate Ikaros activity, a molecular control of lymphocyte development. EMBO J 1996;15:5358-69.

23. Gowda C, Song C, Kapadia M, et al. Regulation of cellular proliferation in acute lymphoblastic leukemia by Casein Kinase II (CK2) and Ikaros. Adv Biol Regul 2017;63:71-80.

24. Han J, Jin R, Zhang M, Guo Q, Zhou F. Ikaros 6 protects acute lymphoblastic leukemia cells against daunorubicin-induced apoptosis by activating the Akt-FoxO1 pathway. J Leukoc Biol 2017;101:675-81.

25. Ezzat S, Yu S, Asa SL. Ikaros isoforms in human pituitary tumors: distinct localization, histone acetylation, and activation of the $5^{\prime}$ fibroblast growth factor receptor-4 promoter. Am J Pathol 2003;163:1177-84. 